\title{
Comparison of bifurcation dynamics of turbulent transport models for the $\mathrm{L}-\mathrm{H}$ transition
}

\author{
W Weymiens, ${ }^{1, \text { a) }}$ S Paquay, ${ }^{2} \mathrm{H}$ J de Blank, ${ }^{2}$ and G M D Hogeweij ${ }^{2}$ \\ ${ }^{1)}$ FOM Institute DIFFER - Dutch Institute for Fundamental Energy Research, Association EURATOM-FOM, \\ PO Box 1207, Nieuwegein, The Netherlands \\ ${ }^{2)}$ Eindhoven University of Technology, Department of Applied Physics, \\ PO Box 503, Eindhoven, The Netherlands
}

\begin{abstract}
In more than three decades a large amount of models and mechanisms have been proposed to describe a very beneficial feature of magnetically confined fusion plasmas: the L-H transition. Bifurcation theory can be used to compare these different models based on their dynamical transition structure. In this paper we employ bifurcation theory to distinguish two fundamentally different descriptions of the interaction between turbulence levels and sheared flows. The analytic bifurcation analysis characterises the parameter space structure of the transition dynamics. Herewith, in these models three dynamically different types of transitions are characterised, sharp transitions, oscillatory transitions and smooth transitions. One of the two models has a very robust transition structure and is therefore more likely to be more accurate for such a robust phenomenon as the L-H transition. The other model needs more fine-tuning to get non-oscillatory transitions. These conclusions from the analytic bifurcation analysis are confirmed by dedicated numerical simulations, with the newly developed code Bifurcator.
\end{abstract}

PACS numbers: 52.25 Fi, 52.25 Xz, 52.55 Dy, 52.55 Fa

\section{INTRODUCTION}

In 1982 the ASDEX team discovered the so-called 'High confinement mode' or H-mode ${ }^{1}$, in which the energy confinement of a magnetically confined fusion plasma becomes, typically, twice as good (compared to the standard 'Low confinement mode' or L-mode). Although this very beneficial, L- to H-mode transition has been seen in most present day tokamaks, there is no consensus on the exact mechanism, or combination of mechanisms, responsible for this L-H transition ${ }^{2}$.

In the last three decades the variety of proposed L-H transition models grew extensively. However, the observation of the $\mathrm{L}-\mathrm{H}$ transition in all tokamaks suggests that the responsible mechanism is very robust. Moreover, the type of dynamics during the transition is very characteristic. Therefore, the transition dynamics and the robustness of all proposed L-H transition models should be investigated. Bifurcation analysis ${ }^{3}$ is a useful tool to check the possible transition dynamics within the model. Moreover, it characterizes the parameter space of these possible transition types, and therewith the sensitivity of the model to parameter changes, i.e. its robustness.

Although there may be different mechanisms determining the growth rate and the saturation mechanism of the turbulence, irrespective of the type of turbulence we restrict our description to the evolution of a radial profile of the turbulent fluctuation level, assuming some average over the fluctuations, consisting of a linear term describing the growth of the turbulence and a nonlinear term describing the saturation mechanism, similar to Refs. ??.

a)w.weymiens@differ.nl
I.e., the turbulence evolution can be described by a differential equation describing the magnitude of the turbulence level, containing two generic terms, one for linear growth rate and one for the non-linear saturation mechanism.

Presently, there is consensus about the influence of sheared $\mathbf{E} \times \mathbf{B}$-flows on turbulence ${ }^{4}$. Microscale simulations show that sheared $\mathbf{E} \times \mathbf{B}$-flows can tear apart the radial extent of turbulent eddies ${ }^{5}$. The $\mathbf{E} \times \mathbf{B}$-flow shear reduction mechanism can then act either on the linear or the nonlinear term of the turbulence evolution equation. These two fundamentally different ways to model the interaction between sheared $\mathbf{E} \times \mathbf{B}$-flows and turbulence are investigated in this paper with the use of bifurcation analysis.

Both descriptions do occur in literature. The investigation of the influence of sheared flows on turbulence was initiated by Biglari in $1990^{6}$. From here, the two separate directions where followed. Firstly, Carreras $^{7-10}$ and Diamond ${ }^{11,12}$ followed an analysis where the flow shear reduces the growth rate of the turbulence. In 2003 the zonal flows were added to this discription ${ }^{13,14}$. Recently, two complementary bifurcation analyses of this model were done by $\mathrm{Dam}^{15}$ and $\mathrm{Zhu}^{16}$. Therefore, the influence of these small scale, spatial and temporal, fluctuations of the $\mathbf{E} \times \mathbf{B}$-flow on the bifurcation structure is omitted in this paper.

The anomalous transport due to the turbulence increases linearly with the fluctuation level as suggested by Refs. ${ }^{17-22}$. According to the nonlinear description the anomalous transport is reduced by the flow shear $\propto\left(1+\alpha \omega_{s}^{2}\right)^{-1}$ where $\omega_{s}$ is the shearing rate which was proposed by Hinton ${ }^{23}$, and has been further investigated in Refs. ${ }^{17,18,24}$ and used in the L-H transition models of Refs. ${ }^{21,25}$.

Recently, a different description of anomalous trans- 
port reduction was introduced by $\mathrm{Miki}^{26}$, where the mean flow shear has a double effect on the transport. Firstly, it reduces the transport coefficient directly, and secondly it reduces the transport indirectly via the suppression of the growth rate of the turbulence. In this paper both effects are considered separately as two different descriptions of the same effect.

Thus, there is consensus that sheared $\mathbf{E} \times \mathbf{B}$-flows reduce the anomalous transport, however there is no consensus yet about the way it should be modelled. Therefore, in this paper two different descriptions of the effects of sheared flows on the transport are compared on the basis of their bifurcation behaviour. In Sec. II the two transport models are introduced. In Sec. III the analytic bifurcation analysis is described. In Sec. IV a numerical code is introduced specially designed for bifurcating systems, and the resulting numerical bifurcation analysis of the considered models is shown in Sec. V.

\section{TURBULENT TRANSPORT MODELS FOR THE L-H TRANSITION}

The degree of confinement is determined by the 1dimensional transport in a tokamak along the minor radius of the torus, i.e. from the hot core of the toroidal plasma towards the colder edge. The radial transport of particles and heat is determined by the particle- and heat- fluxes respectively, in the form of a continuity equation. We consider a layer near the edge of the plasma that is relatively thin compared to the plasma minor radius. We approximate this layer, just for conciseness, with a slab geometry, such that these equations become

$$
\begin{aligned}
\frac{\partial n}{\partial t} & =-\frac{\partial \Gamma}{\partial r} \\
\frac{\partial}{\partial t}\left(\frac{n T}{\gamma-1}\right) & =-\frac{\partial q}{\partial r}
\end{aligned}
$$

where we assume equal temperatures of the ions and electrons, the absence heat sources inside the layer, and that the combination of particle sources, sinks and pinches inside this layer is not drastically changed by the L-H transition. Therefore, the particle and heat fluxes are given by

$$
\begin{aligned}
\Gamma & =-D \frac{\partial n}{\partial r} \\
q & =-\chi n \frac{\partial T}{\partial r}+\frac{\Gamma T}{\gamma-1}
\end{aligned}
$$

The particle flux, $\Gamma$, is governed by some effective particle diffusion due to the anomalous transport of electrons and ions. The heat flux, $q$, is a combination of some effective radial thermal conduction and heat advection due to the net mass-flow described by the particle flux, with $\gamma$ the adiabatic index. A change from low confinement to high confinement can therefore be described by a change in the transport coefficients; particle diffusivity, $D$, and heat conductivity, $\chi$. The minimum amount of transport is determined by neoclassical effects, on top of that anomalous transport depends on the turbulence level. According to Refs. ${ }^{17-22}$ the anomalous transport increases linearly with the turbulence level $\mathcal{E}$,

$$
D=D_{\min }+\left(D_{\max }-D_{\min }\right) \frac{\mathcal{E}}{\mathcal{E}_{\max }}
$$

where $\mathcal{E}_{\text {max }}=\gamma_{L} / \alpha_{\text {sat }}$ is the steady state turbulence level without any flow shear suppression. $\gamma_{L}$ is the linear growth rate of the turbulence and $\alpha_{\text {sat }}$ depends on the saturation mechanism corresponding to that turbulence. Thus, in general the turbulence evolves according to

$$
\frac{\partial \mathcal{E}}{\partial t}=\gamma_{L} \mathcal{E}-\alpha_{\text {sat }} \mathcal{E}^{2}
$$

where the time scale of the turbulence evolution is faster than the diffusive time scale. It is generally accepted ${ }^{2}$ that the turbulence level is reduced by $\mathbf{E} \times \mathbf{B}$-flow shear. This paper addresses the comparison between the two fundamental possibilities in reducing the turbulence. Either, the flow shear reduces the growth rate of the turbulence $^{7-14}$,

$$
\frac{\partial \mathcal{E}}{\partial t}=\gamma_{L}\left(1-\tilde{\alpha}\left|V_{\mathbf{E} \times \mathbf{B}}^{\prime}\right|^{2}\right) \mathcal{E}-\alpha_{s a t} \mathcal{E}^{2},
$$

which is named the linear model in this paper, or it enhances the saturation mechanism of the turbulence which is consistent with the descriptions in Refs. ${ }^{17,18,21,23-25}$,

$$
\frac{\partial \mathcal{E}}{\partial t}=\gamma_{L} \mathcal{E}-\alpha_{\text {sat }}\left(1+\tilde{\alpha}\left|V_{\mathbf{E} \times \mathbf{B}}^{\prime}\right|^{2}\right) \mathcal{E}^{2} .
$$

which is named the nonlinear model in this paper. A radial electric field in a slab geometry causes a poloidal $\mathbf{E} \times \mathbf{B}$-drift, $V_{\mathbf{E} \times \mathbf{B}}=E_{r} / B$, such that the turbulence suppression term can be renormalised, $\tilde{\alpha}\left|V_{\mathbf{E} \times \mathbf{B}}^{\prime}\right|^{2}=\alpha Z^{\prime 2}$, where $Z$ is the normalised radial electric field,

$$
Z=\frac{\rho_{p} e E_{r}}{T_{i}}, \quad \text { and } \quad \rho_{p}=\frac{m v_{t h}}{q B_{p}} .
$$

Since the L-H transition occurs on a very fast time scale, a proper model for the L-H transition should include the evolution of the flow on this fast time scale. The evolution of the radial electric field is determined by the sum of all possible radial currents in the edge of the plasma,

$$
\varepsilon_{0} \frac{\partial E_{r}}{\partial t}=-\sum J_{r}
$$

An extensive list of all the possible mechanisms generating a radial current present in literature is given in Appendix A. All these terms are summarised into an evolution equation for the normalised radial electric field,

$$
\varepsilon \frac{\partial Z}{\partial t}=\mu \frac{\partial^{2} Z}{\partial r^{2}}+c_{n} \frac{T}{n^{2}} \frac{\partial n}{\partial r}+\frac{c_{T}}{n} \frac{\partial T}{\partial r}-G(Z)
$$

where the left-hand side (LHS) includes the polarization current. The right-hand side (RHS) is arranged into a 
second derivative term, the terms depending on the profiles of density and temperature, and all the other terms are incorporated in the large polynomial $G(Z)$, as described in Appendix A. For the description of the transition behaviour of this model it is only required that the function, $G(Z)$, has an inflection point. Since we are only interested in the transition behaviour of this model, we zoom in at this inflection point by taking only the Taylor expansion around this inflection point, $Z=Z_{s}$, into account,

$$
G(Z) \approx a+b\left(Z-Z_{s}\right)+\left(Z-Z_{s}\right)^{3} .
$$

The considered models now consist of a closed set of four coupled nonlinear equations, three partial differential equations (PDEs), Eqs. (1a), (1b) and (9), and one ordinary differential equation (ODE) for the turbulence, Eq. (5) or Eq. (6). The co-ordinate system in which the set of equations will be evaluated is chosen as follows: the outer edge of the plasma at the scrape-off layer (SOL) side is fixed at $x=0$. The inner boundary of the considered spatial domain is located at $x=-\infty$, this is allowed because compared to the size of the transport barrier the inner boundary is far enough away. At this inner boundary all the particles and heat enters the system (there are no additional sources within the domain). These influxes can be used as control parameters of the system:

$$
\begin{aligned}
\Gamma(x=-\infty) & =\text { constant }=\Gamma_{-\infty} \\
q(x=-\infty) & =\text { constant }=q_{-\infty} \\
Z^{\prime}(x=-\infty) & =0
\end{aligned}
$$

At the other boundary of the system, i.e. the outer edge of the plasma, the temperature, density and radial electric field are forced to drop toward zero with a certain efolding length into the scrape-off layer,

$$
\frac{T_{e}^{\prime}}{T_{e}}=\frac{-1}{\lambda_{T}}, \quad \frac{n_{e}^{\prime}}{n_{e}}=\frac{-1}{\lambda_{n}}, \quad \frac{Z_{e}^{\prime}}{Z_{e}}=\frac{-1}{\lambda_{Z}},
$$

with constant gradient lengths $\lambda_{T}, \lambda_{n}$ and $\lambda_{Z}$ of the same order of magnitude, and where from now on the subscript " $e$ " is used for SOL edge values. In the next section the possible transition types within these two models are analysed analytically, and confirmed by numerical bifurcation analysis in Sec. V.

\section{BIFURCATION ANALYSIS}

For the investigation of transition dynamics from one steady state to another it is necessary to characterise the possible steady states. Since the three PDEs of both models are the same, we start by analysing those steady state equations. First of all, we do not expect the L-H transition to be initiated by some specific difference between the two transport coefficients, $D$ and $\chi$. Therefore we can make the following simplification: $\chi=D / \zeta(\gamma-1)$, with $\zeta$ a proportionality factor, leading to the following steady state transport equations:

$$
\begin{aligned}
-D(\mathcal{E}) \frac{\partial n}{\partial r} & =\Gamma_{-\infty} \\
-\frac{D(\mathcal{E})}{\gamma-1}\left(\frac{n}{\zeta} \frac{\partial T}{\partial r}+T \frac{\partial n}{\partial r}\right) & =q_{-\infty}
\end{aligned}
$$

The term proportional to $\mu$ in Eq. (9) smoothens the spatial jump between the L-mode core and the H-mode edge. Since clear identification of the pedestal region is desirable to discriminate L-mode from H-mode, the assumption that $\mu \rightarrow 0$ is used in this analytic bifurcation analysis. In the numerical bifurcation analysis of Sec. V the deviations for small $\mu$ are taken into account. Therefore the radial electric field steady state equation reduces to,

$$
c_{n} \frac{T}{n^{2}} \frac{\partial n}{\partial r}+\frac{c_{T}}{n} \frac{\partial T}{\partial r}-G(Z)=0
$$

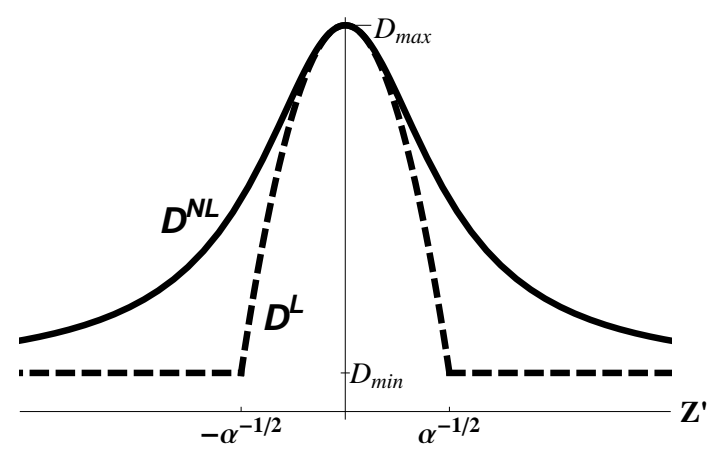

FIG. 1: The transport coefficients as function of the radial electric field shear for

the linear model $(\boldsymbol{L})$, i.e. Eq. (17) for $\left|Z^{\prime}\right|<\sqrt{1 / \alpha}$, and the nonlinear model $(\boldsymbol{N L})$, i.e. Eq. (18).

Combining $^{3}$ the above equations leads to,

$$
-G(Z) D(\mathcal{E})=\frac{T_{-\infty} D\left(\mathcal{E}_{e}\right)^{2}}{\Gamma_{-\infty} \lambda_{n}^{2}}\left(c_{n} \hat{n}^{-2}+c_{g} \hat{n}^{-\zeta-2}\right),
$$

where $\hat{n}=n_{0} / n_{0 e}$, is the steady state density normalised to its edge value, $n_{0 e}=\Gamma_{-\infty} \lambda_{n} / D\left(Z_{e}\right) . T_{-\infty}=(\gamma-$ 1) $q_{-\infty} / \Gamma_{-\infty}$ is the core boundary value of the steady state temperature, and $c_{g}=\left(\zeta c_{T}-c_{n}\right) /\left(1+\zeta \lambda_{T} / \lambda_{n}\right)$. The difference between the two models arises due to the turbulence level ODE. In case of the linear model the steady states are

$$
\begin{cases}\mathcal{E}=0, & \text { stable for } Z^{\prime} \geqslant \sqrt{1 / \alpha} \\ \mathcal{E}=\frac{\gamma_{L}}{\alpha_{\text {sat }}}\left(1-\alpha Z^{\prime 2}\right), & \text { stable for } Z^{\prime}<\sqrt{1 / \alpha}\end{cases}
$$

For the nonlinear model the steady states are,

$$
\left\{\begin{array}{lrl}
\mathcal{E} & =0, & \text { always unstable, } \\
\mathcal{E}=\frac{\gamma_{L}}{\alpha_{\text {sat }}} \frac{1}{1+\alpha Z^{\prime 2}}, & \text { always stable. }
\end{array}\right.
$$


Using these expressions for the turbulence the resulting steady state diffusivities as function of $Z^{\prime}$ of both models can be compared, as is also shown in Fig. 1.

$$
\begin{aligned}
& D^{L}\left(Z^{\prime}\right)=D_{\min }+\left(D_{\max }-D_{\min }\right)\left(1-\alpha Z^{\prime 2}\right), \\
& D^{N L}\left(Z^{\prime}\right)=D_{\min }+\frac{D_{\max }-D_{\min }}{1+\alpha Z^{\prime 2}} .
\end{aligned}
$$

This nonlinear description of the transport reduction is exactly equivalent with what is proposed in Refs. ${ }^{17,18,21,23-25}$. The bifurcation analysis of this nondynamic transport reduction was done in Ref. ${ }^{27}$. Since the steady states of both those models are equal, the bifurcation dynamics of both models are expected to be qualitatively equivalent, as will be shown below.

Evaluating Eq. (14) at the edge boundary, and using the Robin boundary conditions in Eqs. (17) and (18) leads to the following steady state criterion,

$$
D\left(Z_{e}\right)^{2}=-\frac{\Gamma_{-\infty}^{2} \lambda_{n}^{2}}{q_{-\infty}(\gamma-1)} \frac{1}{c_{n}+c_{g}} G\left(Z_{e}\right) D\left(Z_{e}\right),
$$

such that both sides of the equation can be parameterised by $Z_{e}$ as is shown in Fig. 2. The RHS corresponds to the tilted dashed line with a slope,

$$
\theta=\frac{\Gamma_{-\infty}^{2} \lambda_{n}^{2}}{q_{-\infty}(\gamma-1)} \frac{1}{c_{n}+c_{g}}
$$

The LHS is slightly different for both models as is indicated by the two curves $(\boldsymbol{L}$ and $\boldsymbol{N} \boldsymbol{L})$. The intersection between the LHS-curve and the RHS-line indicated with the black dot determines the edge value of the radial electric field, $Z_{e}$, and therewith the transport coefficient at the edge of the plasma corresponding to either L-mode

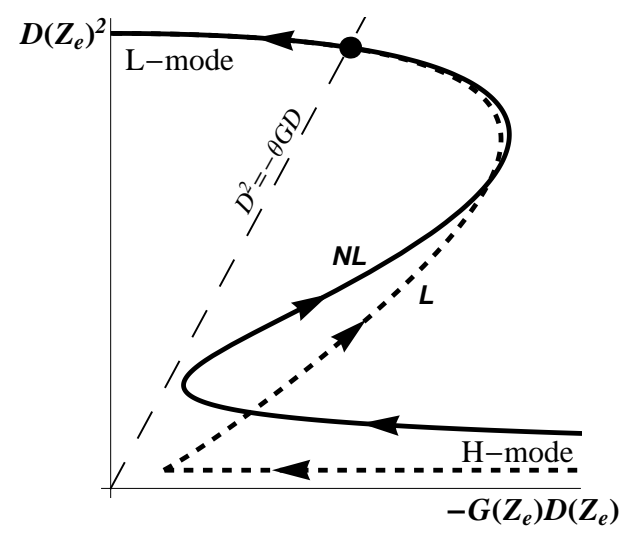

FIG. 2: Both sides of the steady state Eq. (14) can be parameterised by $Z_{e}$. The RHS corresponds to the tilted dashed line. The LHS corresponds to the solid curve for the nonlinear model, and the dashed curve for the linear model, where the arrows indicate increasing $Z_{e}$, and $\alpha=1.0$. The intersection, marked by the black dot, determines the edge steady state. or H-mode. For large $\theta$ (as shown in Fig. 2) the intersection occurs for large diffusivity (squared) at the edge, i.e. L-mode, and for small values of $\theta$ the intersection occurs at small edge diffusivity, i.e. H-mode. The parameter $\theta$ is for instance controlled by the heat flux, $q_{-\infty}$, and is therefore consistent with experiments where an increasing heating power is used to bring the plasma from Lmode to H-mode.

The bifurcation theory introduced in Ref. ${ }^{3}$ showed that the stability of the steady states changes at those values of the edge radial electric field where the slope of the curves, indicated with $\boldsymbol{L}$ and $\boldsymbol{N L}$, are vertical, i.e. the Hopf bifurcation. Thus for decreasing $\theta$ the black dot of Fig. 2, i.e. the steady state $Z_{e}$ solution, moves along the curve (either $\boldsymbol{L}$ or $\boldsymbol{N L}$ ) until those curves have a vertical slope (as indicated in e.g. Fig. 3(a) the right most point for the nonlinear model and Fig. 4(a) for the linear model). At that value of $\theta$ the Hopf bifurcation makes the L-mode unstable. Depending on the stability of the $\mathrm{H}$-mode at that point, two things can happen. If the $\mathrm{H}-$ mode is stable, than the system quickly transits (on the time scale of $\varepsilon$ ) to the stable $\mathrm{H}$-mode. If the $\mathrm{H}$-mode is also unstable (as is shown in Fig. 3b), then the system will oscillate according to a stable limit cycle.

The different possibilities for the nonlinear model are shown in Fig. 3. In part a, the H-mode is stable at the Hopf bifurcation of the L-mode steady state, and therefore sharp transitions will occur. In panel $b$ the $\mathrm{H}$-mode is unstable and therefore the system will follow the limit cycle solution. In panel c the Hopf bifurcations disappeared and the stable L-mode smoothly transits to the stable H-mode. These three different types of transitions (sharp, oscillatory and smooth), are arranged by the parameter $b$ of Eq. (10).

In contrast, for different values of $b$ the transition dynamics (increasing and decreasing $q_{-\infty}$ ) of the linear model does not change. As is shown in Fig. 4 for the same range of values of $b$ as for the nonlinear model, Fig. 3. In all three cases the H-mode is unstable at the point of the Hopf bifurcation of the L-mode, and therefore the system will always have an oscillatory phase during the transition from L-mode to $\mathrm{H}$-mode and back.

These observations about the possible transition dynamics are summarised in Fig. 5 where the parameter spaces $\left(b, q_{-\infty}\right)$ of both models are shown with the Hopf bifurcation curves separating the L-mode, H-mode and oscillatory regimes.

In Fig. 5(a) the parameter space of the nonlinear model is shown. In the middle region the oscillations separate the L-mode from the H-mode. To the left the Hopf bifurcations switch order and sharp transitions with hysteresis arise, because the Hopf bifurcation of the H-mode (i.e. H-L transition) occurs at lower threshold values of the heat flux than the L-H transition. To the right the Hopf bifurcations disappear and the region of L-modes is smoothly connected to the regime with H-modes. This bifurcation structure of the nonlinear model is very robust, i.e. no small changes in all the parameters consid- 

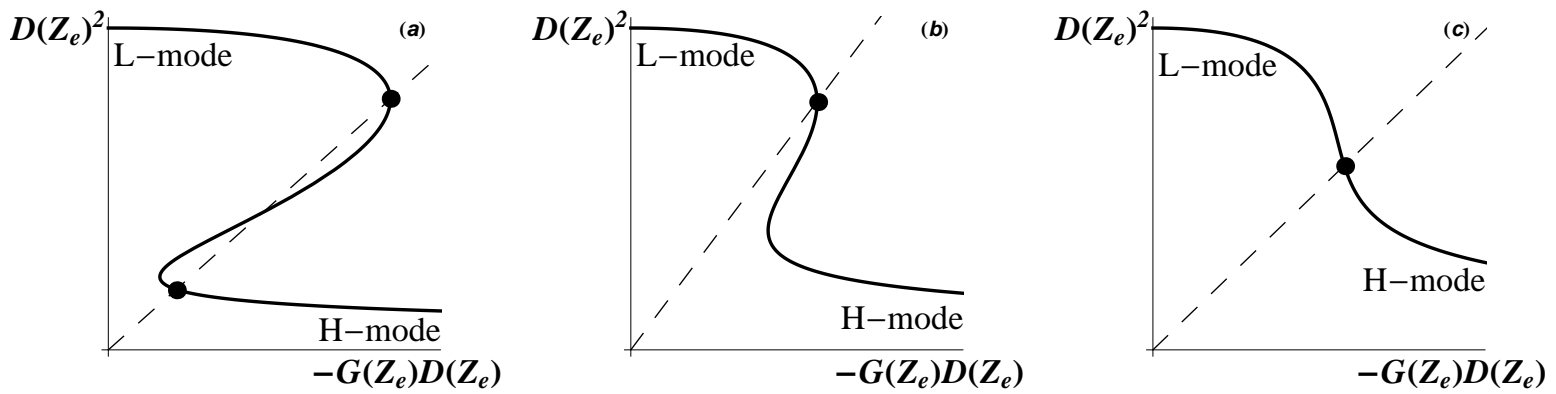

FIG. 3: The edge steady state electric field of the nonlinear model with $\alpha=1.0$ for three different values of $b$. (a) $b=-1.2$, sharp transition between the stable L-mode and the stable H-mode. (b) $b=-0.1$, limit cycle solutions where both L-mode and H-mode are unstable. (c) $b=0.7$, The stable L-mode transits smoothly into a stable H-mode for increasing heat flux.
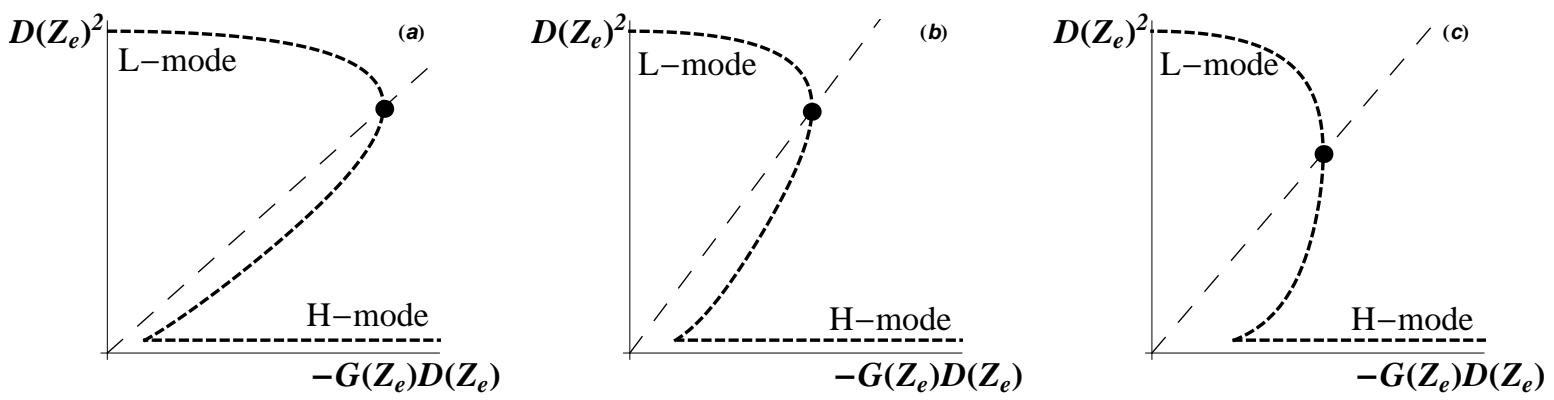

FIG. 4: The edge steady state electric field of the linear model with $\alpha=1.0$ for three different values of $b$. In all three panels there is a regime without stable solutions for decreasing $\theta$ in between the stable L-mode and stable $\mathrm{H}$-mode leading to oscillatory transitions.

ered in this model changes this picture qualitatively. Fig. 5 (b) shows the parameter space of the linear model, and in this case the L-mode and $\mathrm{H}$-mode are always separated by a regime of oscillations. However, the linear model is very sensitive to the parameter $\alpha$. The bifurcation structure changes qualitatively when changing $\alpha$, therefore the possible transition dynamics within this model changes. For $\alpha=1.0$ only oscillatory transitions are possible as is shown in Fig. 5(b), however if $\alpha$ is reduced the $\mathcal{E}=0$ (i.e. $D=D_{\text {min }}$ ) steady state moves further and further away towards higher values of the input heat flux (i.e. smaller $\theta$ ), as is shown in Fig. $6(\alpha=0.1)$. Therefore, the characteristic bifurcation structure reappears again, such that for small $\alpha$ the three different types of transitions (sharp, oscillatory and smooth) are present in this model too, in the characteristic ordering of a codimension 3 bifurcation ${ }^{3}$, as is shown in Fig. 7.

Thus similar transition dynamics can be found in both models. However, in the linear model bifurcation dynamics is very sensitive to $\alpha$. In contrast the nonlinear model is very robust and shows qualitatively the same transition structure for all values of $\alpha$.

To support the conclusions that can be drawn from the analytical bifurcation analysis a dedicated code has been created for the simulation of bifurcating systems of PDEs and/or ODEs, called Bifurcator. The next section gives a short summary of the design of Bifurcator and its capabilities.

\section{BIFURCATOR}

At its core, Bifurcator is a numerical solver for nonlinear ODEs, optimised to meet the accuracy and performance demands of studying bifurcating systems. Bifurcating systems of PDEs, like those describing the L-H transition in fusion plasmas, need to be discretised into a system of ODEs first. While this takes some effort, it allows the user to select the discretisation method most suitable for the problem at hand.

The time integration methods on the other hand are tailored for solving large stiff systems that typically arise from discretising diffusive transport equations like those describing the transport in tokamak edge plasmas. Specifically, Bifurcator employs various implicit RungeKutta (Radau, DIRK and Gauss) methods with an adaptive time stepping strategy to capture all physically relevant time-scales efficiently. Since these methods are implicit, the time integration does involve solving nonlinear systems. This is done using Newton iteration and requires the user to also define the Jacobi-matrix of the discretised system of equations. The adaptive time step 
size is very helpful as the majority of the time the system evolves on a diffusive timescale, while the sudden transitions between metastable states occur in timescales orders of magnitude shorter.

Finally, a bifurcation detection scheme is implemented. In this scheme, Bifurcator obtains the steady state solution for a given set of parameters and varies one parameter over a given interval with some (user-defined) increment. By defining an appropriate "order" parameter for the model, Bifurcator can then detect transitions from one state to another (e.g. from L-mode to H-mode). Whenever such a jump is detected, Bifurcator backtracks to the last solution in the same state as the initial solution, reduces the parameter increment and then again solves the equations at the new parameter value. This allows Bifurcator to approach the critical value of the scanning parameter up to arbitrary accuracy.

All the features mentioned above are modelindependent; the user only needs to supply a discretisation of the equations to be analysed, and optionally an order parameter that describes the transition of interest for the bifurcation detection. At this moment, seven different bifurcating models are already implemented within Bifurcator.

Bifurcator is configured through a combination of input files and command line arguments. These tell the program what model to analyse, which parameters and the time integration settings to use and optionally which parameter to perform a parameter scan in.

The features mentioned above make Bifurcator ideal for studying the models presented in this paper. In the next section the bifurcation detection scheme is used
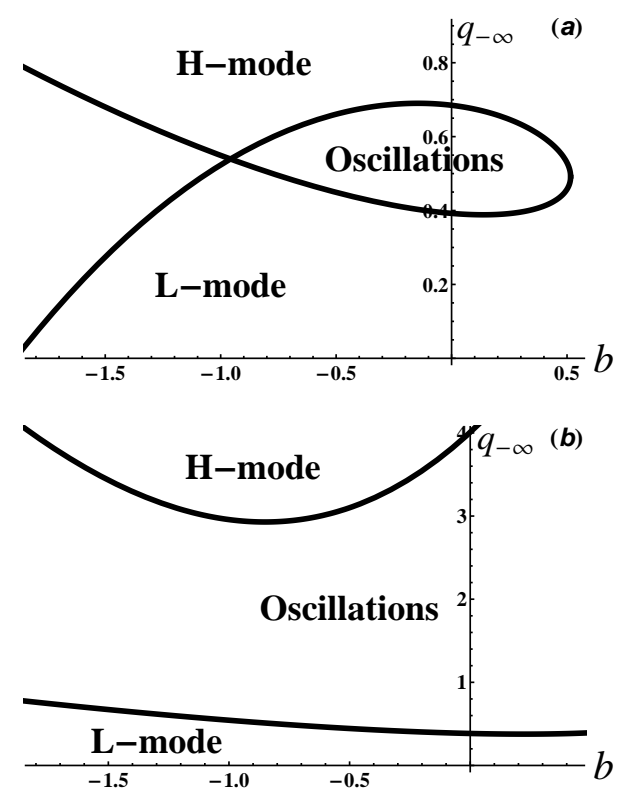

FIG. 5: The Hopf bifurcations for $\alpha=1.0$ in the $\left(b, q_{-\infty}\right)$ parameter space, for (a) the nonlinear model and (b) the linear model.

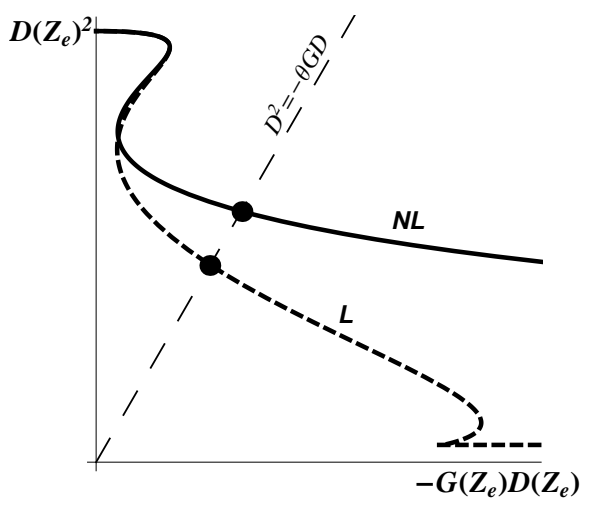

FIG. 6: The radial electric field edge steady state condition for both models with $\alpha=0.1$

to analyse numerically the bifurcating behaviour of the models.

\section{NUMERICAL BIFURCATION ANALYSIS}

The numerical bifurcation analysis consists of many simulations of the considered models during which the heat flux parameter $q_{-\infty}$ is increased (for L-H transitions) or decreased (for $\mathrm{H}-\mathrm{L}$ transitions) in small steps. It is important to simulate every step until the system is in steady state, so no ramping of the heat flux during the simulations, because that could cause additional dynamics. Since the systems are known to exhibit hysteresis it is important during a parameter scan to start every simulation from the steady state profiles of the previous step.

Simulations of the nonlinear model shows L-modes where the radial electric field profile is close to zero everywhere (LHS of Fig. 8) and the turbulence level stays close to $\mathcal{E}_{\max }$, and $\mathrm{H}$-modes where an electric field well is formed near the edge of the plasma (middle of Fig. 8) and locally the turbulence is greatly reduced. Increasing the heat flux, $q_{-\infty}$, in small steps results in L-H transitions that are either sharp (from one step to the next), smooth

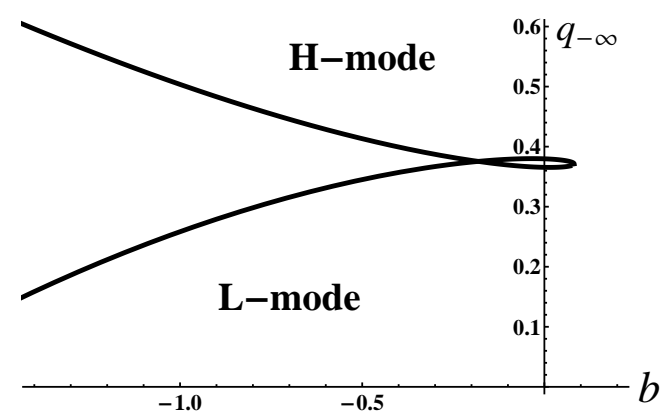

FIG. 7: The bifurcation parameter space of the linear model with $\alpha=0.1$ 
(slowly changing every step), or oscillatory. An example of these oscillations is shown on the RHS of Fig. 8, the radial electric field oscillates covering the shaded area and correspondingly the energy profiles $(U=n T /(\gamma-1))$ oscillate leading to an oscillating outflux, $q_{e}(t)$, for a constant influx, $q_{-\infty}$, as is shown in the inset.

From the bifurcation analysis of the nonlinear model in Sec. III it resulted that the different types of L-H transitions (i.e. sharp, oscillatory and smooth) are ordered by the parameter $b$. This result is confirmed by the Bifurcator simulations shown in Fig. 9. On the right-hand side the series of blue points corresponds to a parameter scan of $q_{-\infty}$ in which the system smoothly went from L-mode profiles to $\mathrm{H}$-mode profiles without any bifurcation. In the intermediate regime of $b$-values the system stays in L-mode up till the value of $q_{-\infty}$ marked by the orange points, increasing the heat flux one additional step and the system enters an oscillatory phase marked by the green points. The system keeps oscillating for increasing heat flux until it reaches a stationary $\mathrm{H}$-mode marked by the red points. In the left most part of the parameter space, the L-mode (orange) and the H-mode (red) are directly adjacent to each other indicating a sharp transition from an L-mode stationary state to an $\mathrm{H}$-mode stationary state for a small increase of the heat flux. Since, in this $b$-parameter range, the back transitions from $\mathrm{H}$-mode to L-mode occur at different values of the heat flux due to the hysteresis, these are separately indicated as the lower branch of red-orange points.

As was noted in Sec. III this nonlinear turbulence model is in steady state exactly the same as the flow shear model analysed in Ref. ${ }^{3}$. Although the dynamical equations are different the same bifurcation structure is therefore expected. Indeed, as is shown in Fig. 10, the bifurcation structure of this model is qualitatively
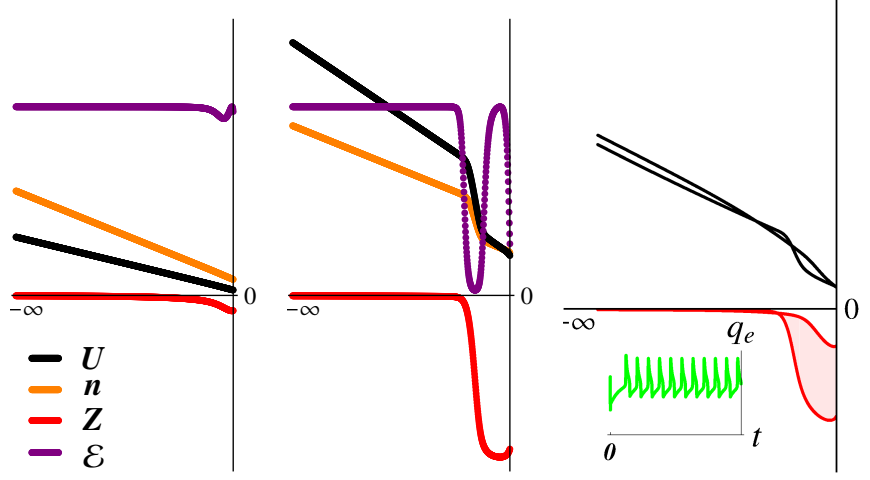

FIG. 8: Three qualitatively different final states of the nonlinear model $(\alpha=1.0)$ i.e from left to right, L-mode, $\mathrm{H}$-mode and the oscillatory phase. Four profiles, energy (black), density (orange), electric field (red), turbulence level (purple) and one time trace of the edge heat flux (green) are indicated. the same. The exact values are somewhat shifted, however the robustness of the bifurcation structure is shown again. The three types of stationary state of this flow shear model without the turbulence dynamics are shown in Fig. 11.

The change from nonlinear reduction of the turbulence by sheared flows to the linear model, does change the bifurcation structure as was shown in Sec. III. The region of oscillations opened up, to all values of $b$, such that only oscillatory transitions from L-mode to $\mathrm{H}$-mode are possible. The numerical parameter scans in the heat flux do indeed show a transition from L-mode into the oscillatory phase for all values of $b$ as is shown in Fig. 12. However, for very high values of the heat flux the profiles do settle in an H-mode steady state that is, however, not fully stationary and keeps oscillating a bit. Since in this part of parameter space we are far away from the organizing center of the co-dimension 3 bifurcation other bifurcations may become visible. Only when $\alpha$ is reduced such that the influence of the sheared flow becomes much less, then the original bifurcation structure corresponding to the co-dimension 3 bifurcation reappears as is shown in Fig. 13 for $\alpha=0.1$. The corresponding profiles of the possible stationary states are shown in Fig. 14.

Thus, the predictions from analytic bifurcation theory are confirmed by dedicated numerical simulations. The exact values of the transitions are slightly different in the numerical simulations than in the analytic analysis. This is mainly due to the assumption of $\mu=0$ in the bifurcation analysis, and the finite value of $\mu=0.05$ in the simulations, because this term directly enters in the equation determining the steady state, Eq. (14). However, the bifurcation structure is very robust and is therefore still

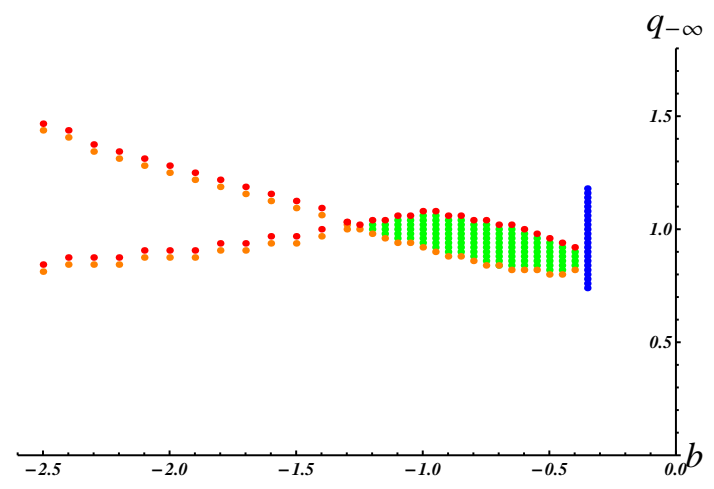

FIG. 9: Simulated parameter space of the nonlinear model $(\alpha=1.0)$, where stable L-modes (orange) and stable H-modes (red) are either adjacent to each other

(left part) indicating sharp transitions, separated by oscillatory phases (green region), or smoothly connected

(blue trace). Note that on the left-hand side two

branches of sharp transition occur, the upper branch corresponds to sharp L-H transitions and the lower one to $\mathrm{H}-\mathrm{L}$ transitions indicating the hysteresis. 


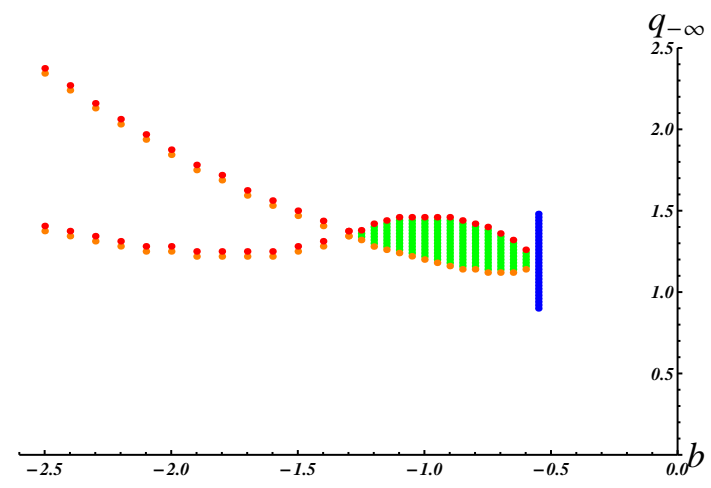

FIG. 10: Numerical bifurcation structure of the flow shear model without turbulence level evolution ${ }^{27}$,

$$
\alpha=1.0
$$
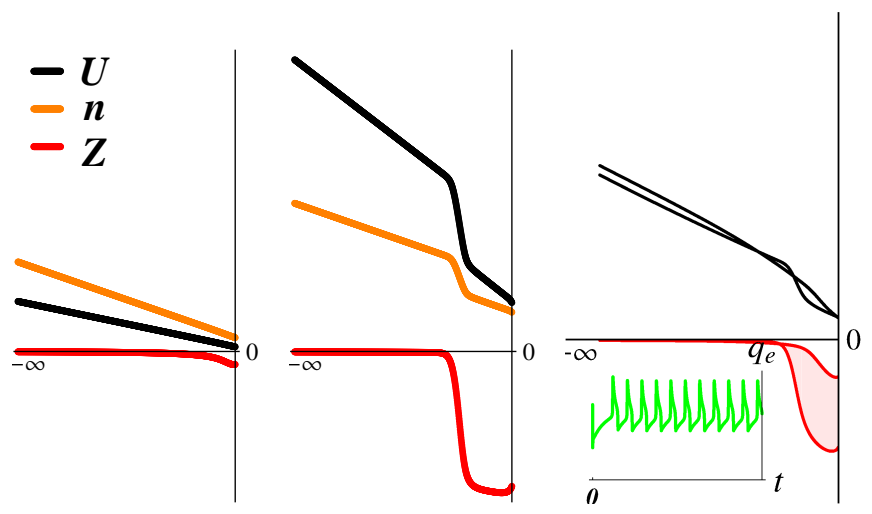

FIG. 11: Three qualitatively different final states of the flow shear reference model without turbulence dynamics $^{27}$. From left to right: L-mode, H-mode and the oscillatory phase. Three profiles, energy (black), density (orange), electric field (red) and one time trace of the edge heat flux (green) are indicated. $(\alpha=1.0)$

intact.

\section{CONCLUSION AND DISCUSSION}

Two fundamentally different descriptions of the turbulence reduction by sheared $\mathbf{E} \times \mathbf{B}$-flows are compared on the basis of their bifurcation structure. The first description reduces the growth rate of the turbulence, and the second description enhances the saturation effect of the turbulence. These two descriptions are incorporated into a transport model evolving the energy, density and radial electric field (and therewith the $\mathbf{E} \times \mathbf{B}$-flow).

On the basis of (analytical and numerical) bifurcation analysis the influence of both turbulence descriptions on $\mathrm{L}-\mathrm{H}$ transition dynamics are compared. The nonlinear model has a very robust bifurcation structure in which

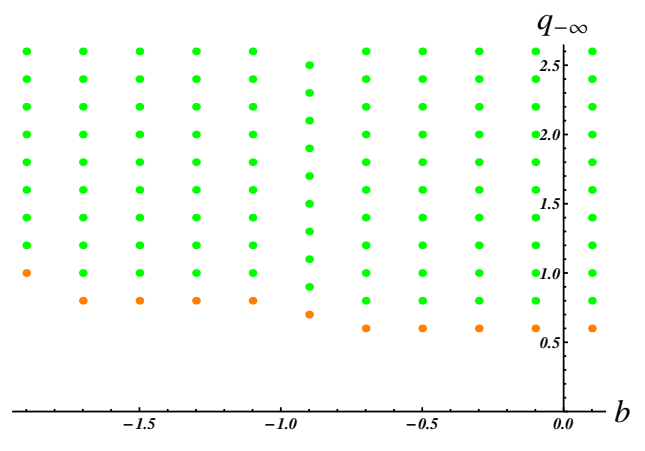

FIG. 12: Numerical bifurcation structure of the linear model for $\alpha=1.0$. Only transitions from L-modes into the oscillatory phase are observed for all values of $b$.

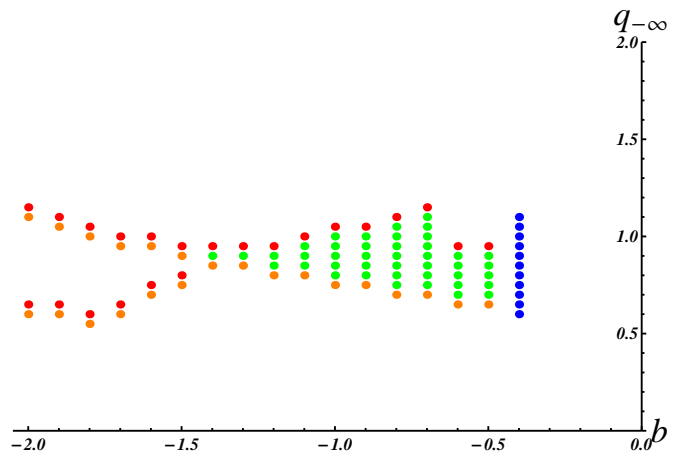

FIG. 13: Numerical bifurcation structure of the linear model for $\alpha=0.1$ corresponds to the characteristic co-dimension $3^{3}$ bifurcation structure.

three different types of transition dynamics can be identified, i.e. sharp, oscillatory and smooth transitions. In contrast, the linear model is sensitive to variations in $\alpha$ (the parameter indicating the effectiveness of the turbulence suppression by sheared flows). Only if this parameter is fine-tuned towards low values the same three types of transition dynamics can be identified. For large values of $\alpha$ the numerical analysis shows only transitions from L-mode to an oscillatory phase. Since the L-H transition is a very robust phenomenon observed in many magnetically confined fusion devices, it is expected that the underlying model is also very robust, which makes the nonlinear model more likely to be the better description.

Another reason why the analysis of transition dynamics is important, is because different types of transition dynamics are observed in experiments, as Carlstrom nicely notes in his review ${ }^{28}$. Most often the transition occurs spontaneously when increasing the heating power, but in low-density discharges at JT-60U ${ }^{29}$ and DIII-D so-called 'transitionless H-modes' were observed. These smooth transitions from L-mode to H-mode without clear bifurcation are also seen in low-density discharges at JET ${ }^{30-32}$, and also 'smoother' transitions are 


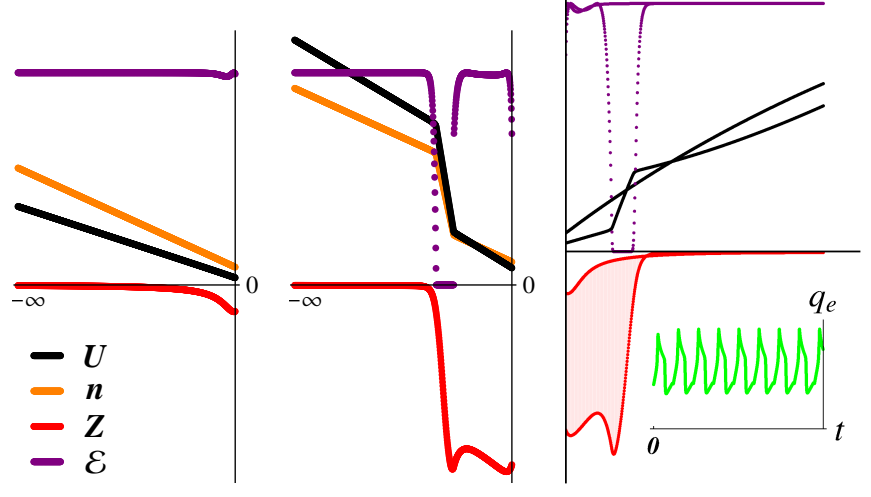

FIG. 14: Three qualitatively different final states of the linear model with $\alpha=0.1$. From left to right: L-mode, H-mode and the oscillatory phase. Four profiles, energy (black), density (orange), electric field (red), turbulence level (purple) and one time trace of the edge heat flux (green) are indicated.

observed at ASDEX Upgrade ${ }^{33}$. Additionally, Carlstrom points out the 'dithering' transitions observed at ASDEX Upgrade $^{34}$, however nowadays there is lots of investigation into all types of oscillatory transitions, at DIII-D ${ }^{35}$, ASDEX Upgrade ${ }^{36,37}$, EAST $^{38,39}$, JET ${ }^{40}$, TJ-II ${ }^{41}$ and HL- $2 \mathrm{~A}^{42}$. Most probably some of these oscillations are caused by the same mechanism, but there could also be multiple mechanisms at play leading to oscillatory behaviour around the L-H transition. Lately, the predatorprey type of oscillations between turbulence and zonal flows is used frequently to describe oscillatory behaviour, as in the papers of Miki ${ }^{26,43-46}$ these type of oscillations are embedded into a similar transport model as discussed in this paper. However, this paper shows that without the zonal flow interaction there are also oscillations inside this model, therefore Miki's model probably has multiple types of oscillations depending on their parameter setting. Therefore, it is essential to do the bifurcation analysis of that model too. As is true for all proposed L-H transition models.

\section{Appendix A: Radial currents}

The evolution of the radial electric field has been subject to debate since the discovery of the H-mode. A large variety of physical mechanisms possibly influencing the radial electric field in magnetically confined plasmas are described in literature. Not one of them has been appointed to be the initiator of the $\mathrm{L}-\mathrm{H}$ transition. Their relative importance to one another is still under discussion. In this appendix a number of radial current drive effects is listed.

$$
\varepsilon_{0} \frac{\partial E_{r}}{\partial t}=-\sum J_{r}=e \sum\left(\Gamma_{e}-\Gamma_{i}\right)
$$

Since, only the dependence on the normalised radial electric field is important for this analysis, we copied the expressions from the relevant literature directly and only indicate the dependence on the normalised radial electric field $Z=\rho_{p} e E_{r} / T_{i}$. First of all, a changing electric field in time causes the generation of a neoclassical polarization current,

$$
J_{p o l}=\frac{\rho c^{2}}{B_{\theta}} \frac{\partial E_{r}}{\partial t}
$$

i.e. Eq. (3) of Ref. ${ }^{47}$, and simulated by Refs. ${ }^{48,49}$. Usually, this term is moved to the LHS of Eq. A1 and combined into the term $\varepsilon \partial Z / \partial t$ of Eq. (9). Furthermore, there are three types of viscosities driving current. Firstly, the shear viscosity,

$$
J_{v i s c}=-\varepsilon_{0} \varepsilon_{\perp} \nabla \cdot \mu_{i} \nabla E_{r}
$$

i.e. Eq. (3) of Ref. ${ }^{50}$, which leads to the term $\mu \partial^{2} Z / \partial r^{2}$ in Eq. (9). This term is also discussed in Ref. ${ }^{51}$ and reviewed in Ref. ${ }^{52}$. Secondly, the bulk viscosity generates a radial current, due to the inhomogeneity of the magnetic field,

$$
\Gamma_{i}^{b v}=f_{b v} \nu_{i} \rho_{p} n_{i} \frac{Z-Z_{0}}{1+Z^{2}}
$$

i.e. Eq. (2.44) from review ${ }^{53}$ and similar to Refs. ${ }^{52,54}$. Initially investigated in Refs. ${ }^{55-57}$. And thirdly, the gyroviscosity generates a radial current according to reviews ${ }^{52,54}$. However, no expression for this effect is given.

Moreover, the anomalous cross field flux has a bipolar part leading to a radial current,

$$
\Gamma_{e}^{a n o m}=-D_{e} n\left(\frac{n^{\prime}}{n}+\alpha \frac{T^{\prime}}{T}+\frac{Z}{\rho_{p}}\right)
$$

i.e. Eq. (3) of Ref. ${ }^{58}$, which initially investigated this effect for the L-H transition. Later it was also used by Ref. $^{57}$, and reviewed in Refs. ${ }^{52-54}$. The first two terms are directly influenced by the density and temperature profiles and are therefore explicitly taken into account in the considered models, see Eq. (9). The third term is absorbed into the function $G(Z)$. A similar expression of current generation is due to the collisional process of ripple diffusion, described in Eq. (19) of Ref. ${ }^{52}$,

$$
\begin{aligned}
\Gamma_{i}^{N C} \simeq-\varepsilon^{2} \sqrt{\varepsilon_{h}} n v_{D}^{2} \int_{0}^{\infty} d w \frac{w^{5 / 2} e^{-w} \nu(w)}{\nu^{2}+\frac{3}{2} \sqrt{\frac{\varepsilon}{\varepsilon_{h}}} w_{r o t}^{2}} \times \\
\left(\frac{n^{\prime}}{n}+(w-3 / 2) \frac{T^{\prime}}{T}-\frac{Z}{\rho_{p}}\right) .
\end{aligned}
$$

Another often proposed radial current generation mechanism is the ion orbit losses or loss cone losses, initially described in Eq. (2) of Ref. ${ }^{51}$,

$$
\Gamma_{i}^{l c}=\frac{1}{\sqrt{\varepsilon}} \frac{n_{i}}{\tau_{i i}} \rho_{p} \hat{F} e^{-Z^{2}} .
$$


and similar expressions are reviewed in Refs. ${ }^{52-54}$. A more general expression for the ion orbit loss rate depending on the collisionality is first given in Ref. ${ }^{59}$, and later used in Eq. (1) of Ref. ${ }^{57}$,

$$
\Gamma_{i}^{l c}=\frac{n_{i} \nu \sqrt{\varepsilon} \rho_{p i}}{\sqrt{\nu_{* i}+Z^{4}}} e^{-\sqrt{\nu_{* i}+Z^{4}}}
$$

In the limit of $\nu_{* i} \rightarrow 0 \mathrm{Eq}$. (A7) is recovered. Furthermore, Reynolds stresses can cause a radial flux of ions, $\Gamma_{i}^{v \nabla v}$, as explained in Ref. ${ }^{52}$. This effect is employed for the L-H transition by Ref. ${ }^{60}$, and reviewed by ${ }^{53,54}$. Moreover, the influx of neutrals and their charge exchange reactions leads to a difference in radial flux of ions compared to electrons, as is firstly used in Ref. ${ }^{61}$, and extensively investigated in Ref. ${ }^{62}$, and reviewed in Ref. $^{52}$ in Eq. (52d),

$$
\Gamma_{i}^{c x}=-n_{0}\left\langle\sigma_{c x} v\right\rangle n_{i} \rho_{p}\left(Z_{0}+Z-q V_{p} / \varepsilon v_{t h}\right) .
$$

Finally, the radial electric field can be manipulated by biasing the plasma with a external voltage ${ }^{50,63}$,

$$
J_{\text {ext }}=\text { constant }
$$

Altogether, this leads to Eq. (9) where the sum of Eqs. (A4)-(A10) is represented by $G(Z)$. For this model to describe L-H transition physics it is necessary that this long polynomial has an inflection point somewhere, and that in the neighbourhood of this point the transport coefficients do vary. Therefore, it is sufficient to take into account only the Taylor expansion around the inflection point to be able to describe L-H transition physics, $G(Z) \approx a+b\left(Z-Z_{s}\right)+\left(Z-Z_{s}\right)^{3}$, similar to Refs. ${ }^{64,65}$. The coefficients $a, b$ and $Z_{s}$ of this Taylor expansion depend, via the different contributions to Eq. (A1) discussed in this appendix, on a large number of quantities that can in principle be determined experimentally, albeit with varying accuracy. Additionally, the influence on the L-H transition by global changes to the plasma, such as triangularity and the direction of the single-null divertor, could also be understood as changes to some of the contributions to Eq. (A1). To determine which parameters can be used as experimental control parameters for the L-H transition, the relative importance of all these effects need to be determined at the edge of the plasma. Since many terms described in this appendix scale with the density, an educated guess could be that the density may be used to control the type of L-H transition.

\section{ACKNOWLEDGEMENTS}

This work, supported by the European Communities under the contract of Association between EURATOM/FOM, was carried out within the framework of the European Fusion Programme with financial support from NWO. The views and opinions expressed herein do not necessarily reflect those of the European Commission. This work is supported by NWO-RFBR Centre-ofExcellence on Fusion Physics and Technology (Grant nr. 047.018.002)

\section{REFERENCES}

${ }^{1}$ Wagner F, Becker G, Behringer K, Campbell D, Eberhagen A, Engelhardt W, Fussmann G, Gehre O, Gernhardt J, v Gierke G, Haas G, Huang M, Karger F, Keilhacker M, Klüber O, Kornherr M, Lackner K, Lisitano G, Lister G G, Mayer H M, Meisel D, Müller E R, Murmann H, Niedermeyer H, Poschenrieder W, Rapp H, Röhr H, Schneider F, Siller G, Speth E, Stäbler A, Steuer K H, Venus G, Vollmer O and Yü Z, Phys. Rev. Lett. 49 1408 (1982)

${ }^{2}$ Wagner F, Plasma Physics and Controlled Fusion 49 B1 (2007)

${ }^{3}$ Weymiens W, de Blank H J, Hogeweij G M D and de Valença J C, Physics of Plasmas 19072309 (2012)

${ }^{4}$ Burrell K H, Physics of Plasmas 64418 (1999)

${ }^{5}$ Burrell K H, Physics of Plasmas 41499 (1997)

${ }^{6}$ Biglari H, Diamond P H and Terry P W, Physics of Fluids B: Plasma Physics 21 (1990)

${ }^{7}$ Carreras B A, Lynch V E, Garcia L and Diamond P H, Physics of Fluids B: Plasma Physics 51491 (1993)

${ }^{8}$ Carreras B A, Diamond P H, Liangs Y M, Lebedev V and Newman D, Plasma Physics and Controlled Fusion 36 A93 (1994)

${ }^{9}$ Carreras B A, Newman D, Diamond P H and Liang Y M, Physics of Plasmas 14014 (1994)

${ }^{10}$ Carreras B A, Lynch V E, Garcia L and Diamond P H, Physics of Plasmas 22744 (1995)

${ }^{11}$ Diamond P H, Shapiro V, shevchenko V, Kim Y B, Rosenbluth M N, Carreras B A, Sidikman K, Lynch V E, Garcia L, Terry P W and Sagdeev R Z, Plasma Physics and Controlled Nuclear Fusion Research 297 (1992)

${ }^{12}$ Diamond P H, Liang Y M, Carreras B A and Terry P W, Phys. Rev. Lett. 722565 (1994)

${ }^{13}$ Kim E-J and Diamond P H , Phys. Rev. Lett. 90185006 (2003)

${ }^{14}$ Kim E-J and Diamond P H, Physics of Plasmas 101698 (2003)

${ }^{15}$ Dam M, Brøns M, Rasmussen J J, Naulin V and Xu G, Physics of Plasmas 20102302 (2013)

${ }^{16}$ Zhu H, Chapman S C and Dendy R O, Physics of Plasmas 20 042302 (2013)

${ }^{17}$ Hahm T S, Physics of Plasmas 12940 (1994)

${ }^{18}$ Hahm T S and Burrell K H, Physics of Plasmas 21648 (1995)

${ }^{19}$ Diamond P H, Lebedev V B, Newman D E and Carreras B A, Physics of Plasmas 23685 (1995)

${ }^{20}$ del Castillo-Negrete D, Carreras B A and Lynch V E, Plasma Physics and Controlled Fusion 46 A105 (2004)

${ }^{21}$ Gürcan Ö D, Diamond P H, Hahm T S and Singh R, Physics of Plasmas 14042306 (2007)

${ }^{22}$ Calvo I and Carreras B A, Physics of Plasmas 14102507 (2007)

${ }^{23}$ Hinton F L, Physics of Fluids B: Plasma Physics 3696 (1991)

${ }^{24}$ Zhang Y Z and Mahajan S M, Physics of Fluids B: Plasma Physics 41385 (1992)

${ }^{25}$ Hinton F L, Staebler G M and Kim Y B, Plasma Physics and Controlled Fusion 36 A273 (1994)

${ }^{26}$ Miki K, Diamond P H, Gürcan Ö D, Tynan G R, Estrada T, Schmitz L and Xu G S, Physics of Plasmas 19092306 (2012)

${ }^{27}$ Weymiens W, de Blank H J and Hogeweij G M D, Physics of Plasmas 20082306 (2013)

${ }^{28}$ Carlstrom T N, Plasma Physics and Controlled Fusion 381149 (1996)

${ }^{29}$ Mori M, Plasma Physics and Controlled Fusion 36 A39 (1994)

${ }^{30}$ Horton L D, Conway G D, Hawkes N C, Lingertat J, Lowry C G, Maggi C F, Righi E, Saibene G, Sartori R, Schunke B, Sips A C C and Start D F H, 26th EPS Conference on Plasma Physics 23J 193 (1999) 
${ }^{31}$ Andrew Y, Sartori R, Righi E, de la Luna E, Hacquin S, Howell D, Hawkes N, Horton L, Huber A, Korotkov A, O'Mullane M and JET-EFDA contributors, Plasma Physics and Controlled Fusion 48479 (2006)

${ }^{32}$ Maggi C, Delabie E, Biewer T, Groth M, Hawkes N, Lehnen M, de la Luna E, McCormick K, Reux C, Rimini F, Solano E, Andrew Y, Bobkov V, Brix M, Calabro G, Czarnecka A, Flanagan J, Lerche E, Marsen S, Nunes I, van Eester D and JET-EFDA Contributors, submitted to Nucl. Fusion, ' $L-H$ power threshold studies in JET with Be/W and C wall' (2013)

${ }^{33}$ Ryter F, Suttrop W, Brüsehaber B, Kaufmann M, Mertens V, Murmann H, Peeters A G, Stober J, Schweinzer J, Zohm H and the ASDEX Upgrade Team, Plasma Physics and Controlled Fusion 40725 (1998)

${ }^{34}$ Zohm H, Suttrop W, Buchl K, de Blank H J, Gruber O, Kallenbach A, Mertens V, Ryter F and Schittenhelm M, Plasma Physics and Controlled Fusion 37437 (1995)

${ }^{35}$ Schmitz L, Zeng L, Rhodes T L, Hillesheim J C, Doyle E J, Groebner R J, Peebles W A, Burrell K H and Wang G, Phys. Rev. Lett. 108155002 (2012)

${ }^{36}$ Conway G D, Angioni C, Ryter F, Sauter P and Vicente J (ASDEX Upgrade Team), Phys. Rev. Lett. 106(6) 065001 (2011)

${ }^{37}$ Ryter F, Rathgeber S, Barrera Orte L, Bernert M, Conway G, Fischer R, Happel T, Kurzan B, McDermott R, Scarabosio A, Suttrop W, Viezzer E, Willensdorfer M, Wolfrum E and the ASDEX Upgrade Team, Nuclear Fusion 53113003 (2013)

${ }^{38}$ Xu G S, Wan B N, Wang H Q, Guo H Y, Zhao H L, Liu A D, Naulin V, Diamond P H, Tynan G R, Xu M, Chen R, Jiang M, Liu P, Yan N, Zhang W, Wang L, Liu S C and Ding S Y, Phys. Rev. Lett. 107125001 (2011)

${ }^{39}$ Wang H, Xu G, Guo H, Wan B, Naulin V, Ding S, Yan N, Zhang W, Wang L, Liu S, Chen R, Shao L, Xiong H, Liu P, Jiang M and Luo G N, Nuclear Fusion 52123011 (2012)

${ }^{40}$ Loarte A, Monk R D, Kukushkin A S, Righi E, Campbell D J, Conway G D and Maggi C F, Phys. Rev. Lett. 833657 (1999)

${ }^{41}$ Estrada T, Hidalgo C, Happel T and Diamond P H, Phys. Rev. Lett. 107245004 (2011)

${ }^{42}$ Cheng J, Dong J Q, Itoh K, Yan L W, Xu M, Zhao K J, Hong W Y, Huang Z H, Ji X Q, Zhong W L, Yu D L, Itoh S I, Nie L, Kong D F, Lan T, Liu A D, Zou X L, Yang Q W, Ding X T, Duan X R and Liu Y (HL-2A Team), Phys. Rev. Lett. 110 $265002(2013)$
${ }^{43}$ Miki K, Diamond P H, Schmitz L, McDonald D C, Estrada T, Gürcan Ö D and Tynan G R, Physics of Plasmas 20062304 (2013)

${ }^{44}$ Miki K, Diamond P, Fedorczak N, Gürcan Ö D, Malkov M, Lee C, Kosuga Y, Tynan G, Xu G, Estrada T, McDonald D, Schmitz L and Zhao K, Nuclear Fusion 53073044 (2013)

${ }^{45}$ Miki K, Diamond P H, Hahn S H, Xiao W W, Gürcan Ö D and Tynan G R, Phys. Rev. Lett. 110195002 (2013)

${ }^{46}$ Miki K, Diamond P H, Hahn S H, Xiao W W, Gürcan Ö D and Tynan G R, Physics of Plasmas 20082304 (2013)

${ }^{47}$ Hinton F L and Robertson J A, Physics of Fluids 271243 (1984)

${ }^{48}$ Shurygin R V and Dewar R L, Plasma Physics and Controlled Fusion 371311 (1995)

${ }^{49}$ Kiviniemi T P, Sipilä S K, Rozhansky V A, Voskoboynikov S P, Kaveeva E G, Heikkinen J A, Coster D P, Schneider R and Bonnin X, Physics of Plasmas 102604 (2003)

${ }^{50}$ Kasuya N, Itoh K and Takase Y, Nuclear Fusion 43244 (2003)

${ }^{51}$ Itoh K, Itoh S I, Yagi M and Fukuyama A, Physics of Plasmas 54121 (1998)

${ }^{52}$ Itoh K and Itoh S I, Plasma Physics and Controlled Fusion 381 (1996)

${ }^{53}$ Connor J W and Wilson H R, Plasma Physics and Controlled Fusion 42 R1 (2000)

${ }^{54}$ Itoh K, Plasma Physics and Controlled Fusion 36 A307 (1994)

${ }^{55}$ Shaing K C and Crume E C, Phys. Rev. Lett. 632369 (1989)

${ }^{56}$ Itoh K and Itoh S I, Nuclear Fusion 322243 (1992)

${ }^{57}$ Toda S, Itoh S I, Yagi M, Fukuyama A and Itoh K, Plasma Physics and Controlled Fusion 381337 (1996)

${ }^{58}$ Itoh S I and Itoh K, Phys. Rev. Lett. 602276 (1988)

${ }^{59}$ Shaing K C, Crume Jr E C and Houlberg W A, Physics of Fluids B: Plasma Physics 21492 (1990)

${ }^{60}$ Hassam A B, Antonsen T M, Drake J F and Liu C S, Phys. Rev. Lett. 66309 (1991)

${ }^{61}$ Itoh S I and Itoh K, Nuclear Fusion 291031 (1989)

${ }^{62}$ D'Ippolito D A and Myra J R, Physics of Plasmas 9853 (2002)

${ }^{63}$ Cornelis J, Sporken R, van Oost G and Weynants R, Nuclear Fusion 34171 (1994)

${ }^{64}$ Itoh S I, Itoh K, Fukuyama A and Miura Y, Phys. Rev. Lett. 67 2485 (1991)

${ }^{65}$ Zohm H, Phys. Rev. Lett. 72222 (1994) 